\title{
A NEW TYPE OF CHARGE SCREENING DUE TO PHONON-COULOMB MIXING IN MANY-BODY PHYSICS
}

\author{
Karl SVOZIL \\ Institut für Theoretische Physik, Technische Universität Wien, Karlsplatz 13/136, A-1040 Vienna, Austria
}

Received 16 July 1984

\begin{abstract}
New forms of charge shielding are obtained by taking into account the mixing of the phonon and Coulomb fields mediated by their electronic polarizability.
\end{abstract}

The calculation of the phonon-electron and electric charge screening is a standard procedure in manyparticle physics ${ }^{\ddagger 1}$. However, very little has been published on its exact form and its field theoretic definition from renormalization techniques [2]. A consistent evaluation of charge screening, as it is done in this letter yields results which cannot be predicted by reasoning based on evidence from classical (not quantized) potential theory alone. The notion that a charge is shielded by the polarization of its surrounding charges of the same type has to be refined when more than one potential is acting between them. An operational measurement of one potential will always be influenced by the other interaction (and vice versa). In particular, the screening of one potential will depend on the screening of the other potential. Subtlety lies in the complex structure of quantum field theory with its various radiative corrections mixing the fields in such a way that it is impossible to redefine the full potential as the bare potential divided by a factor (the dielectric constant), which will be referred to as multiplicative screening. The question then is what is the meaning of this type of charge screening. Renormalization theory gives a satisfactory answer. It is the purpose of this letter to find new insight into these mechanisms of charge shielding.

The lagrangian formalism is chosen as a starting point for further considerations. To describe the dynamics in solids, the lagrangian density of the electron

$\$ 1$ For a treatment of charge screening see e.g. ref. [1]. field $\psi_{s}(x, t)$ ( $s$ is the spin index), the scalar field of the acoustic phonon $\varphi(x, t)$ and the (electrostatic) Coulomb field $\phi(x)$ can be written as [3]:

$L=L_{\mathrm{el}}+L_{\varphi, \phi}+L_{\mathrm{int}}$,

$L_{\mathrm{el}}(\boldsymbol{x}, t)=\sum_{s} \psi_{s}^{\dagger}(\boldsymbol{x}, t)\left\{\mathrm{i} \partial / \partial t-\epsilon_{p}\right\} \psi_{s}(x, t)$,

$L_{\varphi, \phi}(x, t)=\frac{1}{2}[(\mathrm{i} \partial / \partial t, \mathbf{V}) \varphi(x, t)]^{2}+\frac{1}{2}[\mathbf{V} \phi(x)]^{2}$,

$L_{\mathrm{int}}(\boldsymbol{x}, t)=\sum_{s} \psi_{s}^{\dagger}(\boldsymbol{x}, t) \psi_{s}(\boldsymbol{x}, t)[g \varphi(x, t)+e \phi(x)]$.

After quantization, perturbation theory is applied, and the following situation is encountered immediately: since the phonon and Coulomb fields couple to the charge density of the electron, polarization diagrams with respectively one outgoing phonon leg and one outgoing Coulomb leg mix the fields and mediate a transition (at least virtual) between each other [4]. Therefore a comprehensive treatment must be made. It is further assumed that these fields are matrix renormalizable [5]. This means that the following relation between bare fields and full fields (indicated by an asterisk) can in component notation be written as

$\sigma_{i}(x, t)=Z_{i j} \sigma_{j}^{*}(x, t)$,

where we have arranged the fields in vector form $\sigma(x$, $t)=(\varphi(x, t), \phi(x))$. Eq. (2) is the defining equation for the renormalization matrix $Z$. In the special case where the fields are multiplicative renormalizable (which is always assumed without proof in screening 


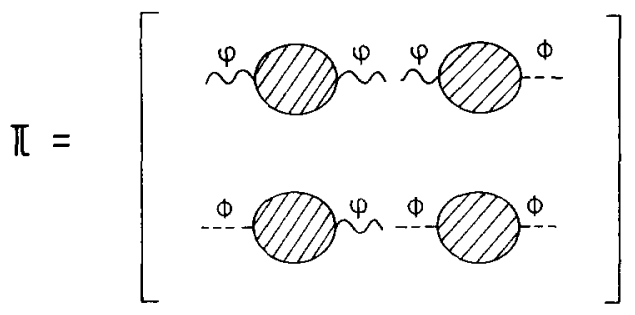

Fig. 1. The irreducible (proper) polarization of the phononCoulomb system.

calculations), the nondiagonal terms of $Z$ vanish.

We now turn to the explicit evaluation of $Z$. From eq. (2) the propagator of the phonon and Coulomb field, defined by $G_{i j}=\left\langle T \sigma_{i} \sigma_{j}\right\rangle$, transforms in matrix notation as ( $\mathrm{t}$ indicates transposition) $G=Z G^{*} Z^{\mathrm{t}}$. For the model lagrangian density (1) the bare propagator in momentum space is of diagonal form $G(q, \omega)$

$=\operatorname{diag}\left(G_{\varphi}(q, \omega), G_{\phi}(q)\right)$ and the irreducible (proper) polarization can be written as (see fig. 1)

$\Pi(q, \omega)=\left[\begin{array}{ll}g^{2} & e g \\ e g & e^{2}\end{array}\right] P(q, \omega)$.

From Dyson's equation for the full propagator $G^{*}-1$ $=G^{-1}-\Pi, G^{*}$ can be computed explicitly $(\operatorname{det}=1$ $\left.-\left\{g^{2} G_{\varphi}+e^{2} G_{\phi}\right\} P\right)$ :

$G^{*}=\operatorname{det}^{-1}\left[\begin{array}{cc}\left(1-e^{2} G_{\phi} P\right) G_{\varphi} & e g G_{\varphi} P G_{\phi} \\ e g G_{\varphi} P G_{\phi} & \left(1-g^{2} G_{\varphi} P\right) G_{\phi}\end{array}\right]$.

The renormalization matrix $Z$ can be evaluated from the scaling law of the propagator. Its components are not uniquely defined and a block form can be assum. ed such that $Z_{21}$ vanishes:

$Z=\left[\begin{array}{cc}\left(1-g^{2} G_{\varphi} P\right)^{1 / 2} & \frac{-e g G_{\varphi} P}{\left(1-g^{2} G_{\varphi} P\right)^{1 / 2}} \\ 0 & \left(\frac{\operatorname{det}}{1-g^{2} G_{\varphi} P}\right)^{1 / 2}\end{array}\right]$

Eq. (4) has two limits:

(i) for such strong-coupling materials where $e \ll g$, $Z$ is given by $Z=\operatorname{diag}\left(\left(1-g^{2} G_{\varphi} P\right)^{1 / 2}, 1\right)$ in agreement with previous results [4], and

(ii) for $e \gg g, Z$ is given by $Z=\operatorname{diag}(1,(1$ $\left.-e^{2} G_{\phi} P\right)^{1 / 2}$, which yields just the usual screening of the Coulomb potential [1]. We now turn to the scaling of the electron field $\psi$, the vertex part $\Gamma$ and the charges The charges, in analogy to the Bose fields, have to be arranged in a vector form $Q=(g, e)^{\mathrm{t}}$. The complete set of Dyson's equations (see refs. $[2,4]$ ) is invariant under the transformation (2) and

$\psi=Z_{\psi} \psi^{*}$,

$\Gamma=\left(Z_{\Gamma}\right)^{-1}$,

$Q=Z_{\Gamma}\left(Z_{\psi}\right)^{-2}\left(Z^{\mathrm{t}}\right)^{-1} Q^{*}$.

For any perturbative calculation this enables us to write the full fields and charges in terms of the bare fields and the screened (renormalized) charges, in which all the scaling factors have been absorbed, thereby taking into account all radiative corrections.

Eq. (5c) defines the screening of the charges. In what follows we assume that $\left(Z_{\psi}\right)^{-2} Z_{\Gamma}$ is of the order of unity $[2,4]$. Due to the block form of $Z$ only $g$ screens multiplicatively: $Z_{11} g=g^{*}$. In the following $g^{*}$ is identified with the coupling constant $g_{0}$ measured from "far away" such that screening effects are negligible. This leads to charge screening of the Feynman-Stueckelberg type (here $\alpha_{g}=g^{2} / 4 \pi$ ):

$\alpha_{g}(q, \omega)=\alpha_{g_{0}} / \epsilon_{g}(q, \omega)$,

$\epsilon_{g}(q, \omega)=1-4 \pi \alpha_{g_{0}} G_{\varphi}(q, \omega) P(q, \omega)$.

From eq. (5c) it can be easily seen that in this scheme of renormalization the screening of the electric coupling constant is of nonmultiplicative character (only reducing to its standard form if $e \gg g$ ) and cannot be written in a closed form as in (6).

Since $\phi(x)$ is independent of $t$, the lagrangian densities $L_{\varphi, \phi}$ and $L_{\text {int }}$ are invariant under an orthogonal rotation of the fields (and the charges) into two new fields $\rho=(\zeta, \xi): \sigma=R_{\theta} \rho$ with

$R_{\theta}=\left(\begin{array}{rr}\cos \theta & \sin \theta \\ -\sin \theta & \cos \theta\end{array}\right)$.

If $\theta=\arctan (e / g)$ the fields in $\rho$ decouple and become multiplicative renormalizable. From eq. (2) the relation between the associated diagonal matrix $Y$ (for $\rho$ ) and $Z$ can be obtained: $Z=R_{-\theta} Y R_{\theta^{*}}$. On equating coefficients one obtains

$Z_{22}=\frac{g^{*}}{g} \frac{\sin \theta \cos \theta}{\sin \theta^{*} \cos \theta^{*}}$. 




Fig. 2. The static dielectric functions $\epsilon_{g}(x, 0)$ and $\epsilon_{h}(x, 0)$ as a function of momentum in units of $2 k_{\mathrm{F}}, \beta=\gamma=1$.

After defining an effective charge $h$ by

$h=\frac{g}{\sin \theta \cos \theta}$

and using the multiplicative scaling of the Coulomb propagator $G_{\phi}=\left(Z_{22}\right)^{2} G_{22}^{*}$ (that is due to the block form of $Z$ ), a multiplicative scaling for $h$ is obtained $\left(\alpha_{h}=h^{2} / 4 \pi\right)$ :

$\alpha_{h}(q, \omega)=\alpha_{h_{0}} / \epsilon_{h}(q, \omega)$,

$\epsilon_{h}(q, \omega)=1-\frac{e^{2} G_{\phi}(q) P(q, \omega)}{1-4 \pi \alpha_{g_{0}} G_{\varphi}(q, \omega) P(q, \omega)}$.

Fig. 2. shows the static dielectric functions $\epsilon_{g}(x, 0)$ and $\epsilon_{h}(x, 0)$ as a function of the momentum in units of twice the Fermi momentum $x=|q| / 2 k_{\mathrm{F}}$. For the proper polarization term its lowest order contribution $P^{(0)}(x, 0)$ (known as the RPA) was inserted $[6](\beta$ $=\left(m_{\mathrm{el}} / \pi^{2}\right)\left(k_{\mathrm{F}} / \omega_{0}^{2}\right) g_{0}^{2}$ and $\left.\gamma=\left(m_{\mathrm{el}} / 2 \pi k_{\mathrm{F}}\right) e^{2}\right)$ $\epsilon_{g}(x, 0)=1+\beta p(x, 0)$.

$\epsilon_{h}(x, 0)=1+\frac{1}{x^{2}} \frac{\gamma p(x, 0)}{1+\beta p(x, 0)}$.

$p(x, 0)=1+\frac{1}{2 x}\left(1-x^{2}\right) \ln \left|\frac{1+x}{1-x}\right|$.

Similarly, the investigation of dynamical screening can be performed by inserting $P^{(0)}(q, \omega)$ into eqs. (6) and (8).

The advantage of the outlined approach is a consistent treatment of the shielding mechanism for charge screening when two or more potentials couple to the same fermionic current. Usually it is impossible to redefine the fields and charges in such a way that their screening is factorizable. For the quite general form of the lagrangian density (1) however, two charges can be defined which, due to the block form of the renormalization matrix, screen multiplicatively.

The author gratefully acknowledges the help he received from Professor O. Hittmair, Dr. H. Nowotny and Dr. R. Haase, and support from the Österreichische Forschungsgemeinschaft during a stay at Berkeley, where he experienced a warm welcome from Professor M.L. Cohen and L. Falicov.

\section{References}

[1] G.D. Mahan, Many-particle physics (Plenum, New York, 1981) pp. 448f, 544-553.

[2] V.L. Bonch-Bruevich and S.V. Tyablikov, The Green function method in statistical mechanics (North-Holland, Amsterdam, 1962) pp. 68-84.

[3] Y. Nambu, Phys. Rev. 117 (1960) 648.

[4] K. Svozil, LBL-preprint 16305 (June 1983), to be published in Phys. Rev. B.

[5] L. Baulieu and R. Coqueraux, Ann. Phys. 140 (1982) 163.

[6] L. Lindhard, K. Dan. Vidensk. Selsk. Mat. Fys. Medd. 28 (1954) No. 8. 